\title{
REMARKS OF GLOBAL WELLPOSEDNESS OF LIQUID CRYSTAL FLOWS AND HEAT FLOWS OF HARMONIC MAPS IN TWO DIMENSIONS
}

\author{
ZHEN LEI, DONG LI, AND XIAOYI ZHANG \\ (Communicated by Walter Craig)
}

\begin{abstract}
We consider the Cauchy problem to the two-dimensional incompressible liquid crystal equation and the heat flows of the harmonic maps equation. Under a natural geometric angle condition, we give a new proof of the global wellposedness of smooth solutions for a class of large initial data in energy space. This result was originally obtained by Ding-Lin and Lin-Lin-Wang. Our main technical tool is a rigidity theorem which gives the coercivity of the harmonic energy under a certain angle condition. Our proof is based on a frequency localization argument combined with the concentration-compactness approach which can be of independent interest.
\end{abstract}

\section{INTRODUCTION}

We consider the following hydrodynamic system modeling the flow of nematic liquid crystal materials in two dimensions (see, for instance, [5, 7, 8]):

$$
\left\{\begin{array}{l}
u_{t}+(u \cdot \nabla) u+\nabla p=\Delta u-\nabla \cdot(\nabla d \otimes \nabla d), \\
d_{t}+(u \cdot \nabla) d=\Delta d+|\nabla d|^{2} d \\
\nabla \cdot u=0, \quad|d|=1
\end{array}\right.
$$

where $u$ is the velocity field, $p$ is the scalar pressure and $d=\left(d_{1}, d_{2}, d_{3}\right)$ is the unit-vector $\left(|d|=\sqrt{d_{1}^{2}+d_{2}^{2}+d_{3}^{2}}=1\right)$ on the sphere $\mathbb{S}^{2} \subset \mathbb{R}^{3}$ representing the macroscopic molecular orientation of the liquid crystal materials. Here the $i^{\text {th }}$ component of $\nabla \cdot(\nabla d \otimes \nabla d)$ is given by $\nabla_{j}\left(\nabla_{i} d \cdot \nabla_{j} d\right)=\sum_{j=1}^{3} \nabla_{j}\left(\nabla_{i} d \cdot \nabla_{j} d\right)$. We apply Einstein's summation convention over repeated indices throughout this paper. For simplicity, we have set all the positive constants in the system to be one. We are interested in the Cauchy problem of (1.1) with the initial data

$$
u(0, x)=u_{0}(x), \quad d(0, x)=d_{0}(x), \quad \lim _{|x| \rightarrow \infty} d_{0}(x)=\hat{n} \in \mathbb{S}^{2},
$$

where $\hat{n}$ is a given unit-vector.

The above system (1.1) is a simplified version of the Ericksen-Leslie model for the hydrodynamics of nematic liquid crystals [5, 7]. The mathematical analysis of the liquid crystal flows was initiated by Lin and Liu in [8, 9. In [15, Wang proved the global existence of strong solutions for rough initial data with sufficiently small $\mathrm{BMO}^{-1}$ norm (for $u_{0}$ and $\nabla d_{0}$ ). See also [10] for a small data global existence result in $3 \mathrm{D}$. For any bounded smooth domain in $\mathbb{R}^{2}$, Lin, Lin and Wang [11] obtained the

Received by the editors May 11, 2012 and, in revised form, October 5, 2012.

2010 Mathematics Subject Classification. Primary 35Q35. 
existence of global weak solutions which are smooth everywhere except on finitely many time slices (see also [6]). The uniqueness of weak solutions in two dimensions was studied in [12,16]. Very recently, a family of non-trivial two-dimensional exact large solutions was constructed in 4 .

In this paper, we are concerned with the global existence of large classical solutions to (1.1). As always with these types of problems, to extend the smooth local solution globally in time one needs to obtain certain a priori estimates. By the regularity theory in 11, 1 the smooth local solution $(u, d)$ to (1.1) can be continued past any time $T>0$ provided that we have

$$
\int_{0}^{T}\left(\|u(t, \cdot)\|_{L^{4}}^{4}+\|\nabla d(t, \cdot)\|_{L^{4}}^{4}\right) d t<\infty .
$$

The basic energy inequality associated with (1.1) is the following:

$$
\begin{gathered}
\frac{1}{2}\left(\|u(t, \cdot)\|_{L^{2}}^{2}+\|\nabla d(t, \cdot)\|_{L^{2}}^{2}\right)+\int_{0}^{t}\left(\|\nabla u(s, \cdot)\|_{L^{2}}^{2}+\left\|\Delta d(s, \cdot)+|\nabla d|^{2} d(s, \cdot)\right\|_{L^{2}}^{2}\right) d s \\
\leq \frac{1}{2}\left(\left\|u_{0}\right\|_{L^{2}}^{2}+\left\|\nabla d_{0}\right\|_{L^{2}}^{2}\right), \quad \forall t \geq 0 .
\end{gathered}
$$

By (1.3)-(1.4) and the Gagliardo-Nirenberg inequality

$$
\|u\|_{L^{4}\left(\mathbb{R}^{2}\right)} \leq C \cdot\|u\|_{L^{2}\left(\mathbb{R}^{2}\right)}^{\frac{1}{2}} \cdot\|\nabla u\|_{L^{2}\left(\mathbb{R}^{2}\right)}^{\frac{1}{2}},
$$

it is obvious that

$$
\begin{aligned}
\|u\|_{L_{t, x}^{4}\left([0, T) \times \mathbb{R}^{2}\right)} & \leq C \cdot\|u\|_{L_{t}^{\infty} L_{x}^{2}\left([0, T) \times \mathbb{R}^{2}\right)}^{\frac{1}{2}} \cdot\|\nabla u\|_{L_{t x}^{2}\left([0, T) \times \mathbb{R}^{2}\right)}^{\frac{1}{2}} \\
& \leq C \cdot\left(\left\|u_{0}\right\|_{L^{2}}+\left\|\nabla d_{0}\right\|_{L^{2}}\right)<\infty, \quad \text { for any } T>0 .
\end{aligned}
$$

Hence the non-blowup criteria (1.3) can be sharpened to

$$
\int_{0}^{T}\|\nabla d(t, \cdot)\|_{L^{4}}^{4} d t<\infty .
$$

Note that in (1.1) if we take $u \equiv 0$ we obtain the usual heat flows of harmonic maps. Condition (1.5) asserts that the regularity of the whole liquid crystal system (1.1) is in some sense governed by the pure heat harmonic flow part. For smooth solutions, the main obstruction in obtaining the a priori estimate (1.5) is that the harmonic energy $E_{\text {harm }}(t)=\left\|\Delta d(t)+|\nabla d(t)|^{2} d(t)\right\|_{2}^{2}$ in (1.4) is not coercive. In particular, it yields no useful lower bounds for general maps $d: \mathbb{R}^{2} \rightarrow \mathbb{S}^{2}$ (a trivial example is the constant map). A natural idea is to introduce some geometric constraints and work with a set of "restricted maps" such that the resulting harmonic energy is coercive. This idea was first used in the seminal work of Ding-Lin in 3 when they were studying a generalization of the Eells-Sampson theorem. Inspired by [3], we follow this line of thought and establish global wellposedness for a family of initial data under a geometric angle condition. Roughly speaking, this condition says that the image of the initial orientation vector $d_{0}$ is strictly contained in a hemisphere. The set of such maps is invariant under the dynamics of (1.1) thanks to the maximum principle.

\footnotetext{
${ }^{1}$ Although the main results in [1] are stated for the bounded domains in $\mathbb{R}^{2}$, it is not difficult to check that the arguments there carry over to the $\mathbb{R}^{2}$ case.
} 
Theorem 1.1. Denote $e_{3}=(0,0,1) \in \mathbb{S}^{2}$. Let $u_{0}, \nabla d_{0} \in L^{2}\left(\mathbb{R}^{2}\right)$ with $\left|d_{0}\right|=1$, $d_{0}-e_{3} \in L^{2}\left(\mathbb{R}^{2}\right)$ satisfy the geometric angle condition

$$
\inf _{x \in \mathbb{R}^{2}} d_{03}>0,
$$

where $d_{03}$ is the third component of $d_{0}$. Then there exists a unique global smooth solution $(d, u)$ to the incompressible liquid crystal equations (1.1) with the initial data (1.2). Moreover, one has

$$
\int_{0}^{\infty}\left(\|\Delta d(t, \cdot)\|_{L^{2}}^{2}+\|\nabla d(t, \cdot)\|_{L^{4}}^{4}+\|\nabla d(t, \cdot)\|_{L^{2}}^{2}\right) d t<\infty .
$$

Remark 1.2. We emphasize that the result in Theorem 1.1 (and also Theorem 1.7 below) can be obtained by using the method of [11.2 Our proof here is based on a frequency localization argument combined with the concentration-compactness approach which can be of independent interest.

Remark 1.3. In Theorem 1.1 the choice of $e_{3}$ is for convenience only. In general one can choose any reference vector $\widehat{n} \in \mathbb{S}^{2}$ and require that the image of $d$ be contained in a hemisphere around $\widehat{n}$. We should also point it out that the geometric angle condition (1.6) may be necessary due to some counterexamples in heat flows of harmonic maps. In [1] the authors proved the global wellposedness of large solutions to the heat flows of harmonic maps for a class of initial data with symmetry:

$$
d_{0}(x)=\left(\begin{array}{c}
x_{1} r^{-1} \sin \psi_{0}(r) \\
x_{2} r^{-1} \sin \psi_{0}(r) \\
\cos \psi_{0}(r)
\end{array}\right),
$$

where

$$
\psi_{0}(0)=0, \psi_{0}(R)<\pi \text { for all } R>0 .
$$

Finite time singularities are also shown to exist in the case where $\psi_{0}(R)>\pi$ for some $R>0$ (see [2] for more details).

Remark 1.4. As was shown in the previous remark, there exists a family of blowup solutions for the heat flows of harmonic maps when the geometric angle condition is violated. These blowup examples furnish a trivial $(u \equiv 0)$ set of counterexamples for the liquid crystal system (1.1). A very interesting open question is whether there are finite time singularities for the incompressible liquid crystal flows in two dimensions with finite energy and non-trivial velocity. We note that such nontrivial counterexamples (if they exist) must be non-radial since the incompressible constraint in the momentum equation of (1.1) destroys the spherical symmetry.

By standard local theory (cf. 11 and 13,14]) there exists a local smooth solution to (1.1) with the initial data (1.2) under the assumptions stated in Theorem 1.1. Moreover, by the weak-strong uniqueness (cf. [12] and [16]), such a solution is also unique. For simplicity of presentation we will refrain from proving such local wellposedness results and only work with smooth local solutions. The main work then is to prove the a priori estimate (1.5). For this we will invoke the geometric angle assumption and prove that the corresponding harmonic energy is coercive. A crucial step is to establish the rigidity of approximate harmonic maps (cf. (2.6)) under the geometric angle condition. We state it as the following:

\footnotetext{
${ }^{2}$ We are grateful to Professor Fanghua Lin for pointing this out to us.
} 
Theorem 1.5. Let $\epsilon_{0}>0, C_{0}>0$. There exists a positive constant $\delta_{0}=\delta_{0}\left(\epsilon_{0}, C_{0}\right)$ such that the following holds:

If $d: \mathbb{R}^{2} \rightarrow \mathbb{S}^{2}, \nabla d \in H^{1}\left(\mathbb{R}^{2}\right)$ with $\|\nabla d\|_{L^{2}} \leq C_{0}$ and $d_{3} \geq \epsilon_{0}$, then

$$
\|\nabla d\|_{L^{4}}^{4} \leq\left(1-\delta_{0}\right)\|\Delta d\|_{L^{2}}^{2} .
$$

Consequently for such maps the associated harmonic energy is coercive, i.e.

$$
\left\|\Delta d+|\nabla d|^{2} d\right\|_{L^{2}}^{2} \geq \frac{\delta_{0}}{2}\left(\|\Delta d\|_{L^{2}}^{2}+\|\nabla d\|_{L^{4}}^{4}\right) .
$$

Remark 1.6. Applying the method of $[3$ one can even obtain a stronger rigidity theorem. We thank Professor Fanghua Lin for pointing this out. Our approach here is based on frequency localization combined with concentration-compactness.

Note that our rigidity Theorem 1.5 is purely "static" and has nothing to do with the velocity field of the fluid dynamics. As such it is stated for any map $d: \mathbb{R}^{2} \rightarrow \mathbb{S}^{2}$ under general assumptions. By using essentially the same arguments, we also have a similar result for the heat flows of harmonic maps:

$$
d_{t}=\Delta d+|\nabla d|^{2} d
$$

where $d$ is still a unit-vector on the sphere $\mathbb{S}^{2} \subset \mathbb{R}^{3}$ (see 3 for a generalization of Eells-Sampson's theorem). We state the result as the following:

Theorem 1.7. Let $\nabla d_{0} \in L^{2}\left(\mathbb{R}^{2}\right)$ with $\left|d_{0}\right|=1, d_{0}-e_{3} \in L^{2}\left(\mathbb{R}^{2}\right)$ and $\inf _{x \in \mathbb{R}^{2}} d_{03}$ $>0$. Then there exists a unique global smooth solution to the heat flows of harmonic maps (1.9) with the initial data $d(0, x)=d_{0}(x)$. Moreover, (1.7) is satisfied.

The proof of Theorem 1.7 will be omitted since it is essentially a repetition of the proof of Theorem 1.1

We close this introduction by setting up some notation and conventions which will be used in this paper.

Notation. For any two quantities $A$ and $B$, we use $A \lesssim B$ (resp. $A \gtrsim B$ ) to denote the inequality $A \leq C B$ (resp. $A \geq C B$ ) for a generic positive constant $C$. The dependence of $C$ on other parameters or constants is usually clear from the context, and we will often suppress this dependence. The value of $C$ may change from line to line. For any function $f: \mathbb{R}^{2} \rightarrow \mathbb{R}$, we use $\|f\|_{L^{p}}$ or sometimes $\|f\|_{p}$ to denote the usual Lebesgue $L^{p}$ norm of a function for $1 \leq p \leq \infty$. We use $L_{t}^{p} L_{x}^{r}$ to denote the space-time norm

$$
\|f\|_{L_{t}^{p} L_{x}^{r}}=\left(\int_{\mathbb{R}}\left(\int_{\mathbb{R}^{2}}|f(t, x)|^{r} d x\right)^{\frac{p}{r}}\right)^{\frac{1}{p}},
$$

with the usual modifications when $p$ or $r$ is infinity, or when the domain $\mathbb{R} \times \mathbb{R}^{2}$ is replaced by a space-time slab. When $p=r$ we abbreviate $L_{t}^{p} L_{x}^{r}$ by $L_{t, x}^{p}$ or $L_{t x}^{p}$.

Occasionally we will need to use the fractional Laplacian operator $|\nabla|^{s}, s>0$, which is defined via Fourier transform as

$$
\mathcal{F}\left(|\nabla|^{s} f\right)(\xi)=|\xi|^{s}(\mathcal{F} f)(\xi), \quad \xi \in \mathbb{R}^{2} .
$$

The homogeneous Sobolev norm $\dot{H}^{s}$ for any $s>0$ is defined as $\|f\|_{\dot{H}^{s}}=\left\||\nabla|^{s} f\right\|_{2}$ or, more explicitly,

$$
\|f\|_{\dot{H}^{s}}=\left(\int_{\mathbb{R}^{2}}|\xi|^{2 s}|(\mathcal{F} f)(\xi)|^{2} d \xi\right)^{\frac{1}{2}}
$$


We will need to use the Littlewood-Paley (LP) frequency projection operators. For simplicity we shall fix the notation on $\mathbb{R}^{2}$, but it is straightforward to define everything in $\mathbb{R}^{d}$ for any $d \geq 1$. To fix the notation let $\phi \in C_{0}^{\infty}\left(\mathbb{R}^{2}\right)$ and satisfy

$$
0 \leq \phi \leq 1, \quad \phi(x)=1 \text { for }|x| \leq 1, \quad \phi(x)=0 \text { for }|x| \geq 2 .
$$

For two real positive numbers $\alpha<\beta$, define the frequency localized (LP) projection operator $P_{\alpha<\cdot<\beta}$ by

$$
P_{\alpha<\cdot<\beta} f=\mathcal{F}^{-1}\left(\left[\phi\left(\beta^{-1} \xi\right)-\phi\left(\alpha^{-1} \xi\right)\right] \mathcal{F}(f)\right) .
$$

Here $\mathcal{F}$ and $\mathcal{F}^{-1}$ denote the Fourier transform and its inverse transform, respectively. Similarly, the operators $P_{<\alpha}$ and $P_{>\beta}$ are defined by

$$
P_{<\beta} f=\mathcal{F}^{-1}\left(\phi\left(\beta^{-1} \xi\right) \mathcal{F}(f)\right)
$$

and

$$
P_{>\alpha} f=\mathcal{F}^{-1}\left(\left[1-\phi\left(\alpha^{-1} \xi\right)\right] \mathcal{F}(f)\right) .
$$

We recall the following Bernstein estimates: for any $1 \leq p \leq q \leq \infty$ and dyadic $N>0$,

$$
\left\|P_{<N} f\right\|_{L_{x}^{q}\left(\mathbb{R}^{2}\right)} \lesssim N^{\frac{2}{p}-\frac{2}{q}}\|f\|_{L_{x}^{p}\left(\mathbb{R}^{2}\right)}
$$

\section{RIGIDITY}

In this section we prove our rigidity Theorem 1.5. Our proof is purely analytic and uses in a quantitative way the geometric angle condition. The key ingredients of the proof are a frequency localization argument and a concentration-compactness procedure.

We begin with the following simple lemma which locks the non-trivial $L^{2}$ weak limit of an $L^{2}$ bounded sequence of functions whose frequency is essentially localized to a (large) ring. The proof is stated for $\mathbb{R}^{2}$, but it naturally generalizes to $\mathbb{R}^{d}$ for any dimension $d \geq 1$.

Lemma 2.1 (Non-evacuation of $L^{2}$-norm). Let $C_{0}$ and $\alpha$ be two positive constants, and $N$ be a dyadic integer. Suppose that $f_{n}: \mathbb{R}^{2} \rightarrow \mathbb{R}$ is a sequence of functions such that

$$
\left\|f_{n}\right\|_{L^{2}} \leq C_{0}, \quad \forall n
$$

and

$$
\left\|P_{\frac{1}{N}<\cdot<N} f_{n}\right\|_{L^{\infty}} \geq \alpha>0, \quad \forall n .
$$

Then one can find a subsequence (which we still label as $f_{n}$ ) and centers $x_{n} \in \mathbb{R}^{2}$ such that

$$
f_{n}\left(x_{n}-\cdot\right) \rightarrow f \quad \text { weakly in } L^{2}
$$

and

$$
\|f\|_{L^{2}} \gtrsim 1
$$

Proof. By (2.1), we can find $x_{n} \in \mathbb{R}^{2}$ such that

$$
\left|\left(P_{\frac{1}{N}<\cdot<N} f_{n}\right)\left(x_{n}\right)\right| \geq \frac{\alpha}{2}, \quad \forall n .
$$


Or more precisely, for $\psi=\mathcal{F}^{-1}\left(\phi\left(N^{-1} \xi\right)-f(N \xi)\right) \in \mathcal{S}\left(\mathbb{R}^{2}\right)$, one has

$$
\left|\int_{\mathbb{R}^{2}} \psi(y) f_{n}\left(x_{n}-y\right) d y\right| \geq \frac{\alpha}{2}, \quad \forall n .
$$

Since by assumption the sequence $f_{n}\left(x_{n}-\cdot\right)$ is uniformly bounded in $L^{2}$, there exists a subsequence (we still denote it by $f_{n}\left(x_{n}-\cdot\right)$ ) which converges weakly in $L^{2}$ to some function $f$. Clearly, by taking the limit in (2.2) and using the CauchySchwartz inequality, one concludes that $\|f\|_{L^{2}} \gtrsim 1$.

Our next lemma allows us to remove the translational degrees of freedom in studying the rigidity property of approximate harmonic maps from $\mathbb{R}^{2}$ to $\mathbb{S}^{2}$ under the geometric angle condition. By removing the translational degrees of freedom (and quotienting out other possible non-compact group actions), we can restore the compactness in the same spirit as the usual concentration-compactness procedure.

Lemma 2.2 (Removing translational invariance). Let $C_{0}$ and $\alpha$ be two positive constants. Suppose a sequence of maps $d_{n}: \mathbb{R}^{2} \rightarrow \mathbb{S}^{2}, n \geq 1$, satisfies the following conditions:

- $\left\|\nabla d_{n}\right\|_{L^{2}}+\left\|\nabla d_{n}\right\|_{\dot{H}^{1}} \leq C_{0}<\infty, \quad \forall n$,

- $\left\|\nabla d_{n}\right\|_{L^{4}}^{2} \geq \alpha>0, \quad \forall n$.

Then, upon a subsequence in $n$ if necessary, we can find a sequence of points $x_{n} \in \mathbb{R}^{2}$ such that

$$
\left|\nabla d_{n}\left(x_{n}-\cdot\right)\right|^{2} \rightarrow f, \quad \text { weakly in } L^{2}
$$

and $\|f\|_{L^{2}} \gtrsim 1$.

Proof. Let $N$ be a dyadic integer which will be taken sufficiently large in the course of the proof. Our main idea is to localize the $\nabla d_{n}$-sequence within the frequency window $[1 / N, N]$. After that we apply Lemma 2.1 to find the non-trivial weak limit.

We first deal with the high frequency piece. By frequency localization, we have

$$
P_{>N}\left(\left(\nabla d_{n}\right)^{2}\right)=P_{>N}\left(P_{>N / 8} \nabla d_{n} \cdot P_{>N / 8} \nabla d_{n}\right)+2 P_{>N}\left(P_{\leq N / 8} \nabla d_{n} \cdot P_{>N / 8} \nabla d_{n}\right) .
$$

By Sobolev embedding, we then have

$$
\begin{aligned}
\left\|P_{>N}\left(\left(\nabla d_{n}\right)^{2}\right)\right\|_{L^{2}} & \lesssim\left\|P_{>N / 8} \nabla d_{n}\right\|_{L^{4}}\left\|\nabla d_{n}\right\|_{L^{4}} \\
& \lesssim\left\||\nabla|^{\frac{1}{2}} P_{>N / 8} \nabla d_{n}\right\|_{L^{2}} \cdot C_{0} \\
& \lesssim N^{-1 / 2}\left\|\Delta d_{n}\right\|_{L^{2}} \cdot C_{0} \\
& \lesssim N^{-1 / 2} C_{0}^{2} \leq \alpha / 10,
\end{aligned}
$$

if we take $N$ large enough.

Similarly for the low frequency part, we use Bernstein's inequality to get

$$
\left\|P_{<\frac{1}{N}}\left(\left(\nabla d_{n}\right)^{2}\right)\right\|_{L^{2}} \leq N^{-1}\left\|\left(\left(\nabla d_{n}\right)^{2}\right)\right\|_{L^{1}} \leq \alpha / 10
$$

where again we need to take $N$ large enough.

Now using (2.3) and (2.4) and the assumption $\left\|\left(\nabla d_{n}\right)^{2}\right\|_{L^{2}} \geq \alpha$, we obtain

$$
\left\|P_{\frac{1}{N}<\cdot<N}\left(\left(\nabla d_{n}\right)^{2}\right)\right\|_{L^{2}} \geq \frac{\alpha}{2}
$$


On the other hand, by Hölder, one has

$$
\begin{aligned}
& \left\|P_{\frac{1}{N}<\cdot<N}\left(\left(\nabla d_{n}\right)^{2}\right)\right\|_{L^{2}} \\
& \lesssim\left\|\left(\nabla d_{n}\right)^{2}\right\|_{L^{1}}^{\frac{1}{2}}\|\| P_{\frac{1}{N}<\cdot<N}\left(\left(\nabla d_{n}\right)^{2}\right) \|_{L^{\infty}}^{\frac{1}{2}} \\
& \lesssim\left\|P_{\frac{1}{N}<\cdot<N}\left(\left(\nabla d_{n}\right)^{2}\right)\right\|_{L^{\infty}}^{\frac{1}{2}} .
\end{aligned}
$$

Obviously

$$
\left\|P_{\frac{1}{N}<\cdot<N}\left(\left(\nabla d_{n}\right)^{2}\right)\right\|_{L^{\infty}} \gtrsim 1 .
$$

We can then apply Lemma 2.1 to the sequence $f_{n}=\left|\nabla d_{n}\right|^{2}$ to get the result.

Now we are ready to prove the rigidity Theorem 1.5 under the geometric angle condition $d_{3} \geq \epsilon_{0}$.

Proof of Theorem 1.5. First of all, using a scaling argument $d(x) \rightarrow d(\lambda x)$ with $\lambda=\|\Delta d\|_{L^{2}}^{-1}$, we may assume

$$
\|\Delta d\|_{L^{2}}=1
$$

It then suffices to show that there exists $\delta_{0}=\delta_{0}\left(\epsilon_{0}, C_{0}\right)>0$ such that

$$
\|\nabla d\|_{L^{4}}^{4} \leq 1-\delta_{0} .
$$

Assume (2.5) does not hold. Then we can find a sequence $d_{n}: \mathbb{R}^{2} \rightarrow \mathbb{S}^{2}$ such that

$$
\left\|\Delta d_{n}\right\|_{L^{2}}=1, \quad\left\|\nabla d_{n}\right\|_{L^{2}} \leq C_{0}, \quad d_{n 3} \geq \epsilon_{0},
$$

but

$$
\left\|\nabla d_{n}\right\|_{L^{4}} \nearrow 1, \text { as } n \rightarrow \infty
$$

Denote

$$
g_{n}=\Delta d_{n}+\left|\nabla d_{n}\right|^{2} d_{n} .
$$

It is easy to compute that

$$
\begin{aligned}
\left\|g_{n}\right\|_{L^{2}}^{2} & =\left\|\Delta d_{n}\right\|_{L^{2}}^{2}+\left\|\nabla d_{n}\right\|_{L^{4}}^{4}+2 \int\left|\nabla d_{n}\right|^{2} \Delta d_{n} \cdot d_{n} d x \\
& =1-\left\|\nabla d_{n}\right\|_{L^{4}}^{4} .
\end{aligned}
$$

Hence

$$
\left\|g_{n}\right\|_{L^{2}} \searrow 0 \quad \text { as } n \rightarrow \infty .
$$

Applying Lemma 2.2 and performing a simple transform to eliminate the translation invariance if necessary, we conclude that

$$
\left|\nabla d_{n}\right|^{2} \rightarrow g(x), \quad \text { weakly in } \mathrm{L}^{2} \text { as } n \rightarrow \infty
$$

for some $g(x) \geq 0$ and $\|g\|_{L^{2}} \gtrsim 1$. Consequently, by taking the limit $n \rightarrow \infty$ for the equation of $d_{n 3}$ in (2.6), we have

$$
\Delta d_{3}^{*}+g(x) \epsilon_{0} \leq 0
$$

where $d_{3}^{*}$ is the weak limit of the sequence (upon subsequence if necessary) $d_{n 3}$ which satisfies

$$
\left\|\Delta d_{3}^{*}\right\|_{L^{2}} \leq 1, \quad\left\|\nabla d_{3}^{*}\right\|_{L^{2}} \leq C_{0} .
$$

Now $d_{3}^{*}$ is superharmonic on $\mathbb{R}^{2}$ and is bounded. Obviously $d_{3}^{*}$ must be a constant map. But since $\Delta d_{3}^{*}+g(x) \epsilon_{0} \leq 0$, one concludes that $g(x)$ must be identically 0 . This contradicts the fact that $\|g\|_{L^{2}} \gtrsim 1$. 
We have obtained the desired contradiction, and therefore (2.5) holds. Finally, (1.8) follows from (2.5) and the same computation (with $d_{n}$ replaced by $d$ ) as in (2.6) and (2.7).

This concludes the proof of Theorem 1.5 .

\section{Proof of Theorem 1.1}

As was already mentioned in the introduction, we only need to establish the a priori estimate (1.5). By using the maximum principle (applied to the third component of $d_{3}$ ), we have

$$
\inf _{x \in \mathbb{R}^{2}} d_{3}(t, x) \geq \inf _{x \in \mathbb{R}^{2}} d_{03}, \quad \forall t>0 .
$$

One can apply the rigidity Theorem 1.5 to conclude that

$$
\left\|\Delta d(t)+|\nabla d(t)|^{2} d(t)\right\|_{L^{2}}^{2} \geq \frac{\delta_{0}}{2}\left(\|\Delta d(t)\|_{L^{2}}^{2}+\|\nabla d(t)\|_{4}^{4}\right), \quad \forall t>0,
$$

where $\delta_{0}>0$ is the same constant as in Theorem 1.5.

Consequently, the basic energy law (1.4) yields

$$
\begin{gathered}
\frac{1}{2}\|\nabla d(t)\|_{L^{2}}^{2}+\frac{\delta_{0}}{2} \int_{0}^{T}\left(\|\Delta d(t)\|_{L^{2}}^{2}+\|\nabla d(t)\|_{L^{4}}^{4}\right) d t \\
\leq \frac{1}{2}\left(\left\|u_{0}\right\|_{L^{2}}^{2}+\left\|\nabla d_{0}\right\|_{L^{2}}^{2}\right), \quad \forall t \geq 0 .
\end{gathered}
$$

This clearly settles (1.5) and the first two terms in the estimate (1.7). It remains for us to prove the estimate

$$
\int_{0}^{\infty}\|\nabla d(t)\|_{L^{2}}^{2} d t<\infty
$$

By performing the basic energy estimate for the equation of $d-e_{3}$ and using the interpolation inequalities, we have

$$
\begin{aligned}
& \frac{1}{2} \frac{d}{d t}\left\|d-e_{3}\right\|_{L^{2}}^{2}+\|\nabla d\|_{L^{2}}^{2} \\
& \leq \int|\nabla d|^{2} d\left(d-e_{3}\right) d x \leq\|\nabla d\|_{L^{4}}^{2}\left\|d-e_{3}\right\|_{L^{2}} \\
& \lesssim\|\nabla d\|_{L^{2}}\|\Delta d\|_{L^{2}}\left\|d-e_{3}\right\|_{L^{2}} \\
& \leq \frac{1}{2}\|\nabla d\|_{L^{2}}^{2}+C\|\Delta d\|_{L^{2}}^{2}\left\|d-e_{3}\right\|_{L^{2}}^{2},
\end{aligned}
$$

where $C>0$ is an absolute constant. A Gronwall in time argument then gives $\left\|d(t, \cdot)-e_{3}\right\|_{L^{2}}^{2}+\int_{0}^{t}\|\nabla d\|_{L^{2}}^{2} d s \lesssim\left\|d_{0}-e_{3}\right\|_{L^{2}}^{2} \exp \left(\int_{0}^{t}\|\Delta d\|_{L^{2}}^{2} d s\right), \quad \forall t \geq 0$.

Noting (3.2), one concludes that

$$
\int_{0}^{t}\|\nabla d\|_{L^{2}}^{2} d s<\infty, \quad \forall t \geq 0 .
$$

This obviously implies (3.3).

This concludes the proof of Theorem 1.1. Finally, we point out that a further higher order energy estimate also implies that any $H^{s}$ norm of $(u, \nabla d)$ is uniformly bounded in time for any $s>0$. 


\section{ACKNOWLEDGEMENTS}

The authors would like to thank Professor Hongjie Dong and Professor Fanghua Lin for many helpful discussions. This work was done when the second and third authors were visiting the School of Mathematical Sciences of Fudan University during 2011. They would like to thank the school for its hospitality.

The first author was supported by the NSFC (grants No. 11171072, 11121101 and 11222107), NCET-12-0120, Innovation Program of Shanghai Municipal Education Commission (grant No. 12ZZ012), Shanghai Talent Development Fund and SGST 09DZ2272900 2.

The second author was supported in part by NSF grant No. 0908032 and in part by the NSF under agreement DMS-1128155. Any opinions, findings and conclusions or recommendations expressed in this material are those of the authors and do not necessarily reflect the views of the National Science Foundation.

The third author was supported in part by an Alfred Sloan Fellowship.

\section{RefEREnCES}

[1] Kung-Ching Chang and Wei Yue Ding, A result on the global existence for heat flows of harmonic maps from $D^{2}$ into $S^{2}$, Nematics (Orsay, 1990), NATO Adv. Sci. Inst. Ser. C Math. Phys. Sci., vol. 332, Kluwer Acad. Publ., Dordrecht, 1991, pp. 37-47. MR1178085 (94c:58048)

[2] Kung-Ching Chang, Wei Yue Ding, and Rugang Ye, Finite-time blow-up of the heat flow of harmonic maps from surfaces, J. Differential Geom. 36 (1992), no. 2, 507-515. MR1180392 (93h:58043)

[3] Wei Yue Ding and Fang-Hua Lin, A generalization of Eells-Sampson's theorem, J. Partial Differential Equations 5 (1992), no. 4, 13-22. MR1192714 (93j:58032)

[4] H. J. Dong and Z. Lei, On a family of exact solutions to the incompressible liquid crystals in two dimensions, preprint.

[5] J. L. Ericksen, Hydrostatic theory of liquid crystals, Arch. Rational Mech. Anal. 9 (1962), 371-378. MR0137403 (25 \#855)

[6] Min-Chun Hong, Global existence of solutions of the simplified Ericksen-Leslie system in dimension two, Calc. Var. Partial Differential Equations 40 (2011), no. 1-2, 15-36, DOI 10.1007/s00526-010-0331-5. MR2745194(2012b:35271)

[7] F. M. Leslie, Some constitutive equations for liquid crystals, Arch. Rational Mech. Anal. 28 (1968), no. 4, 265-283, DOI 10.1007/BF00251810. MR1553506

[8] Fang-Hua Lin and Chun Liu, Nonparabolic dissipative systems modeling the flow of liquid crystals, Comm. Pure Appl. Math. 48 (1995), no. 5, 501-537, DOI 10.1002/cpa.3160480503. MR.1329830 (96a:35154)

[9] Fang-Hua Lin and Chun Liu, Partial regularity of the dynamic system modeling the flow of liquid crystals, Discrete Contin. Dynam. Systems 2 (1996), no. 1, 1-22. MR1367385 (96m:35255)

[10] Xiaoli Li and Dehua Wang, Global solution to the incompressible flow of liquid crystals, J. Differential Equations 252 (2012), no. 1, 745-767, DOI 10.1016/j.jde.2011.08.045. MR2852225 (2012k:35419)

[11] Fanghua Lin, Junyu Lin, and Changyou Wang, Liquid crystal flows in two dimensions, Arch. Ration. Mech. Anal. 197 (2010), no. 1, 297-336, DOI 10.1007/s00205-009-0278-x. MR2646822(2011c:35411)

[12] Fanghua Lin and Changyou Wang, On the uniqueness of heat flow of harmonic maps and hydrodynamic flow of nematic liquid crystals, Chin. Ann. Math. Ser. B 31 (2010), no. 6, 921-938, DOI 10.1007/s11401-010-0612-5. MR2745211 (2011k:58021)

[13] J. Sacks and K. Uhlenbeck, The existence of minimal immersions of 2-spheres, Ann. of Math. (2) 113 (1981), no. 1, 1-24, DOI 10.2307/1971131. MR604040 (82f:58035)

[14] Michael Struwe, On the evolution of harmonic mappings of Riemannian surfaces, Comment. Math. Helv. 60 (1985), no. 4, 558-581, DOI 10.1007/BF02567432. MR826871(87e:58056) 
[15] Changyou Wang, Well-posedness for the heat flow of harmonic maps and the liquid crystal flow with rough initial data, Arch. Ration. Mech. Anal. 200 (2011), no. 1, 1-19, DOI 10.1007/s00205-010-0343-5. MR2781584

[16] Xiang $\mathrm{Xu}$ and Zhifei Zhang, Global regularity and uniqueness of weak solution for the 2-D liquid crystal flows, J. Differential Equations 252 (2012), no. 2, 1169-1181, DOI 10.1016/j.jde.2011.08.028. MR2853534(2012k:35441)

School of Mathematical Sciences, LMnS, and Shanghai Key Laboratory for Contemporary Applied Mathematics, Fudan University, Shanghai 200433, People's Republic OF CHINA

E-mail address: leizhn@gmail.com

Department of Mathematics, University of British Columbia, 1984 Mathematical Road, Vancouver, BC V6T 1Z2, CAnada

E-mail address: mpdongli@gmail.com

Department of Mathematics, 14 Maclean Hall, University of Iowa, Iowa City, Iowa 52242

E-mail address: zh.xiaoyi@gmail.com 